\title{
Distribution of human metapneumovirus among children presenting with acute respiratory tract infection to a General Hospital in Sri Lanka
}

\author{
MVM Divarathna $^{1}$, RAM Rafeek ${ }^{1}$, AMSB Abeykoon ${ }^{1}$, AJ Morel $^{2}$ and F Noordeen ${ }^{1}$
}

\section{Introduction}

Acute respiratory tract infections (ARTIs) are one of the major causes of morbidity and mortality among children under five years. Bacteria, fungi and viruses cause ARTI but viral respiratory infections (VRIs) are the most common diseases in children. Among the viral causes of VRIs, human metapneumovirus (hMPV) was recognized as a common respiratory pathogen after respiratory syncytial virus (RSV). This study aims were to identify the associated factors, distribution and disease burden of hMPV associated VRIs in the present study sample.

\section{Methods}

In this study, we collected demographic and clinical data and nasopharyngeal aspirate (NPA) samples from children with suspected ARTI admitted to the paediatric wards of General Hospital, Kegalle from March 2016 to July 2018. The study sample was children less than 5 years of age with $\leq 4$ days history of ARTI. Viral RNA from the NPA samples was extracted using QIAamp Viral RNA Mini Kit (Qiagen, Germany). A segment of 170bp of the L gene encoding the RNAdirected RNA polymerase L protein of hMPV prototype was amplified using a single step RTPCR. Reverse transcription was carried out using the one step RT-PCR kit (Qiagen, Germany) followed by a PCR using the primers targeting the L gene (L7 and L6).

\section{Results}

From a total of 500 hospitalized children, hMPV was detected in $23.8 \%$ (119/500) by the RT-PCR. Male sex $(\mathrm{M}: \mathrm{F}=1.9: 1)$ and first year of life $(65.5 \%)$ appear to be associated with acquiring hMPV infection in the study sample and $74.8 \%$ (89/119) hMPV positive children had mild to severe bronchiolitis.

\section{Conclusion}

hMPV contributes to the VRIs in $24 \%$ of the children less than 5 years. Less than 12 months of age and male sex appear to be associated with a high number of hMPV associated VRIs including bronchiolitis in the study sample.

Keywords: Viral respiratory infections, human metapneumovirus, epidemiology, children, Sri Lanka

Funding : Research Grant No - NSF/SCH/2017/01 - National Science Foundation of Sri Lanka.

\footnotetext{
${ }^{1}$ Department of Microbiology, Faculty of Medicine, University of Peradeniya, Sri Lanka

${ }^{2}$ General Hospital, Kegalle, Sri Lanka

Address for correspondence: Prof F. Noordeen. Telephone: +94772293301

Email: faseeha.noordeen12@gmail.com iD https://orcid.org/0000-0002-2018-0606
} 